\title{
Design Of Fuzzy Rule-Based System Using Genetic Algorithms
}

\author{
*Sanjay Charaya ${ }^{1}$, Kapil Mehta ${ }^{2}$ \\ ${ }^{1}$ Assistant Professor, Department of Electrical Engineering, Ch. Devi Lal State Institute of Engineering and \\ Technology, VPO-PanniwalaMota, Sirsa, Haryana-125077, India. \\ sanjaycharaya1@gmail.com \\ ${ }^{2}$ Assistant Professor, Department of Computer Science and Engineering, Chandigarh Group of Colleges, \\ Landran, Mohali, Punjab, India. \\ , Kapil.4110@cgc.edu.in
}

\begin{abstract}
The aim of this paper is to obtain a compact and optimal fuzzy rule-based model from observation data by utilizing the Genetic algorithm technique. The approach is optimized by applying Genetic Algorithms, owing to its capability of searching irregular and high dimensional solution spaces. Genetic Algorithms has been applied to learn consequent part of fuzzy rules and models with fixed number of rules.

In the work we propose a Genetic algorithm approach to a non-linear air conditioning system for the construction of optimal fuzzy rules in two steps. First, fuzzy clustering is applied to obtain an initial rule based model having pre-calculated number of rules with antecedents only. In the second step, the regions of rule-consequents are obtained by a binary coded Genetic Algorithm which leads to the extraction of an optimal rule based model.
\end{abstract}

Key words: Genetic Algorithm (GA), Fuzzy Rule Based System (FRBS), Fuzzy set, membership function, membership values, Mamdani-type fuzzy system, Fuzzy rules, Rule Base (RB).

\section{INTRODUCTION}

\section{A. Introduction}

The concept of fuzzy set [1] and fuzzy logic [2] was introduced by the Azerbaijani scientist L.A. Zadeh in mid-1970. Since then a lot of development have taken place in this field. These concepts have been successfully utilized from consumer products and electronic equipment to automobiles and traffic monitoring systems.

In mathematics, sets have been classified as fuzzy set and crisp set. Fuzzy sets give a flexible sense of membership to its elements. While in crisp sets, an element either belongs to (0) or does not belong to (1) a set, in fuzzy set many membership values, between 0 and 1 , are allowed. Similarly, logic can also be viewed as crisp logic and fuzzy logic in the same way as the sets were classified to crisp sets and fuzzy sets. Just as the crisp sets are associated with two states of membership either 0 or 1 and fuzzy sets on multiple membership values, between 0 and 1, crisp logic is built on two states of either 'true' or 'false' and fuzzy logic on a multiple value of true/partly true/very true/false/partly false/very false and so on. By utilizing the fuzzy logic, rules in the form of 'if-then' have been developed for a system. The collection of these rules is called a fuzzy rule base and a system described by a fuzzy rule base is known as 'Fuzzy rule based system' (FRBS) or simply a fuzzy system.

Fuzzy systems describe the complicated systems with linguistic descriptions, which is a simpler and mere human approach to control system design. This feature of fuzzy systems is effective to describe the conditions like cold, warm, very warm, low pressure, high etc. used in everyday life. Further, the conventional control systems require mathematical modelling for its design and it becomes difficult as the system complexities increases. The issue does not arise for fuzzy systems as it does not require mathematical modelling. So, the fuzzy systems are simpler and effective than the conventional control systems.

The designing of an optimal fuzzy system can be considered as equivalent to a search problem in high-dimensional space where each point represents a rule set, membership functions and the corresponding system's behaviour. First the fuzzy systems which satisfy the given performance criteria are found, then, among infinite solution systems, so obtained, the optimal fuzzy system is designed. Due to this genetic algorithm is a better candidate for searching in high-dimensional space than conventional methods.

B. Previous Approaches:

The generation of the fuzzy if-then rules, defining membership function and tuning of the scaling factors are the necessary steps in designing any fuzzy system. The generation of fuzzy rules is accomplished by mainly two types of approaches.

(1) Fuzzy rules are directly designed by the experts of the field.

(2) To generate through an automatic learning process.

The cost of hiring an expert may be high, and also, it is not sure that human expert is always available to make the decision. The first method is then limited and when it is not suitable, an alternative for making a good decision is to utilize automatic learning approach. In the past many 
fuzzy systems that automatically derive fuzzy ifthen rules from the numeric data have been developed.

Wang and Mendel [3] presented a general method to generate fuzzy rules by combining both numerical and linguistic information. The fuzzy rule base so obtained comprises of some rules created by experts and others generated from measured numerical data. Orthogonal transform and fuzzy clustering is utilized to extract fuzzy rules from data in [4]. In [5] on the basis of correlation coefficient threshold value $(\zeta)$, the boundary shift value $(\varepsilon)$ and the center shift value $(\delta)$ the membership functions are constructed and fuzzy rules are generated from training instances. Castro et. al [6] suggested an algorithm to generate fuzzy rules with minimal structure with respect to the number of variables that must appear in the rule and later presented an automatic procedure to generate rules having maximal structure in the sense of both having less components in the antecedent part of the rule and identifying simultaneously the largest number of examples in the given set of input-output data [7]. A general learning method for automatically deriving both membership functions and fuzzy rules to build a fuzzy expert system [8], which is further improved by first selecting relevant attributes and building appropriate initial membership functions [9].

Genetic algorithms are computer based search and optimization algorithms based on the process of natural selection. Prof. J. Holland introduced the concept of these algorithms which was further extended by De Jong, Goldberg, et al. [10], [11].

Depending on the fuzzy control rules, either Mamdani type or TSK type of fuzzy control rule structures has been designed using GA. Table-I shows an example of the Mamdani type of fuzzy control rules with two inputs and single output. For this type of control rules both antecedent and consequent part of a rule are described by a fuzzy set such as low, very low (v.low), extremely low (e. low), medium (med), high, very high (v. high) and extremely high (e. high). Whereas, in TSK type of rule structure the consequent part of the fuzzy rule are replaced by a linear equation of the inputs such as, $a_{0}+a_{0} x_{1}+a_{2} x_{2}+\ldots \ldots \ldots . .+a_{n} x_{n}$,

Table 1

Mamdani Type Fuzzy control rules

\begin{tabular}{|c|c|c|c|c|c|c|c|}
\hline $\boldsymbol{x}_{1} \longrightarrow$ & e. low & v.low & low & med & high & v. high & e. high \\
\cline { 1 - 5 } $\boldsymbol{x}_{\mathbf{2}} \downarrow$ & & & & & & & \\
\hline e.low & med & low & low & v.low & v.low & e.low & e.low \\
\hline v.low & high & med & low & low & v.low & v.low & e.low \\
\hline low & high & high & med & low & low & v.low & v.low \\
\hline med & v.high & high & high & med & low & low & v.low \\
\hline high & v.high & v.high & high & high & med & low & low \\
\hline v. high & e.high & v.high & v.high & high & high & med & low \\
\hline e. high & e.high & e. high & v.high & v.high & high & high & med \\
\hline
\end{tabular}

where $x_{i}$, denotes the ith input with coefficient $a_{i}$.

Karr has used a GA to generate only membership functions for a $\mathrm{pH}$ control process [12] and the cart pole problem [13]. It is possible to design both membership function and rule set using
GA's [14]. It has been done serially, e.g., design the membership function and then use them in the design of rule set. Yuhui Shi et al. presented a GA based method [15], in which the membership function shapes and types and the fuzzy rule set including the number of rules inside it are evolved.

The aim of this article is to develop a compact and optimal fuzzy rule based system (FRBS) for a non-linear air conditioning system using Genetic algorithm (GA). The next section explains about $\mathrm{GA}$ and its basic operations of reproduction, crossover and mutation. Mamdani-type fuzzy rule based model has been used in this paper. Section III describes about fuzzy control systems and its working. The GA based fuzzy logic control procedure adopted is explained in section IV. In section $\mathrm{V}$, we apply this approach to a non-linear air conditioning system. The example results of section VI demonstrate that the method discussed in this paper is effective and efficient. Finally, some concluding remarks are given in section VII.

\section{GENETIC ALGORITHMS: AN OVERVIEW}

The purpose of Genetic Algorithms (GAs) in a problem is to evolve a group of artificial elements called a population and to select possible candidate solutions in a population referred as an individual. A single bit in an individual is called a gene. GA works in the following way; Initially a large no. of individuals are generated randomly. Then the fitness function value of every individual is evaluated. Fitness function value is derived from the objective function of the problem. It is a measure of individual's fitness.GA process is based on the principle of Darwinian theory of survival of the fittest in its search process. According to this principle, only the fittest individuals are retained and rest are discarded. Only the best individuals (parents) are used to produce new offsprings so that the fitness value increases from generation to generation. To ensure that good individuals have more chances for being reproduced, parents are selected with a probability proportional to their relative fitness. This process is called Reproduction. It is to be clear that reproduction also called selection operator creates good individuals but does not create new points in the search space. To create new search points or new individuals, GAs uses mutation and crossover operators. In Crossover operation, two individuals are randomly selected for mating. Then the parts of the parent individuals are swapped to produce new individuals. After crossover the individuals are subjected to mutation. Cross over operation can produce new individuals but still it cannot always create the optimal solution while mutation could. Mutation operator has the ability to modify the basic building block called 
gene of an individual. It maintains diversity in the population.

The implementation of almost all types of GA can be summarized as;

1) Encoding of individuals

2) Generating an initial population of individuals

3) Define the fitness value function and calculate its value the for each individual in population

4) Selection of chromosomes from the population to be parents to crossover.

5) Apply crossover and mutation operators.

6) Go to step-2 subject to certain criterion otherwise terminate the GA.

Encoding and Initialization: In order to use GA unknown variables are first coded in some string structure. There are different methods to code individual genes like binary encoding, octal encoding, hexadecimal encoding, permutation encoding etc. An initial population of individuals are generated at random. Fitness function is defined and evaluated for every individual in population. Fitness function gives direction to the search in problem space.

Reproduction: It is the first operator to be applied on the population. Every chromosome in the population is evaluated for its fitness value. Based upon the merit of fitness values, the chromosomes from the population are selected to be parent for crossover. Roulette-wheel selection, Boltzmann selection, Tournament selection, Rank selection, Steady-state selection etc. are various methods reported for selecting chromosomes for parents to crossover.

Crossover: Reproduction phase selects the population with better individuals for parents to crossover. Crossover operation is applied to the mating pair to create better string. Crossover operation takes place as; First, the mating pair for parent is selected by reproduction operator. The crossover point is randomly chosen, on which parents exchange their genes. For instance, let the selected chromosomes for the mating pair be $\mathrm{X}=$ 11111111 and $\mathrm{Y}=00000000$ and $\mathrm{X}^{\prime}, \mathrm{Y}^{\prime}$ be their children. Further let us choose the crossover point is two, and then we have;

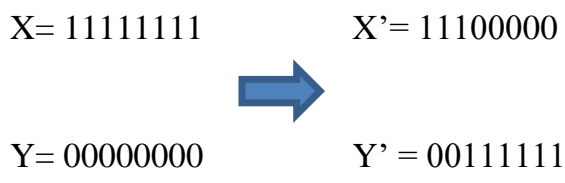

Mutation: Mutation is performed bit-by-bit on a string. It involves flipping of a bit i.e. changing its value from 0 to 1 or vice versa with a specific predefined mutation probability. The mutation of a bit is independent of the probability of mutation for other bits in the string. Mutation can produce individuals in those areas of the search space, where crossover could not regenerate. It leads the algorithm to achieve the global minima in the search space instead to trap in local minima. For example, consider the following population of strings;

$$
\begin{aligned}
& 1100110110 \\
& 0110101100 \\
& 1101011010 \\
& 1001000100
\end{aligned}
$$

The population consists of four ten bit strings and all the string has a zero in their rightmost bit position. Suppose that the optimal solution of the problem has one in their rightmost bit position and neither crossover nor reproduction operations could not create the individuals with one at the rightmost bit position. Then mutation can create, because it has always some probability to change the rightmost bit from zero to one as;

$$
\begin{aligned}
& 1100110110 \\
& 0110101100 \\
& 1101011010 \\
& 1001000101
\end{aligned}
$$

Mutation has the ability to change the basic building blocks called genes of an individual. Hence it can produce that information which earlier was not able to create in the population.

\section{FUZZY CONTROL SYSTEMS}

Mamdani-type of fuzzy rule structure has been followed for the design of air conditioning system model example in section $\mathrm{V}$, whose general form in a fuzzy system is;

If $x_{1}$ is $A_{1}$ and $x_{2}$ is $A_{2}, \ldots \ldots \ldots$, and $x_{k}$ is $A_{k}$ then $y_{1}$ is
$B_{1}$ and $y_{2}$ is $B_{2}, \ldots \ldots .$. , and $y_{k}$ is $B_{k}$ where each $y_{i}$ denotes the consequent (output) variable whose value is inferred, each $x_{i}$ denotes an input variable (an antecedent) and each $A_{i}$ and $B_{i}$ is a fuzzy set represented by a membership function.

Fuzzy rule set and the membership functions associated with its fuzzy sets are the fundamental components of a fuzzy control system. If these are defined, then the fuzzy control system is also defined. Only that function, whose shape closely resembles the values of variables of the physical system, is selected as membership function for that variable. Generally triangular, Gaussian or Sigmoid functions are followed as the membership functions.

The working of fuzzy control system is summarized in series of steps as;

1) From each data set compute, the fuzzy membership values corresponding to each input.

2) Establish the rule in linguistic form with antecedents only.

3) Apply the AND (min.) operator to combine the membership values for each rule. 
4) Compute membership values corresponding to each output in respective fuzzy sets.

5) Apply the OR (minimum) operator to combine the membership values in a fuzzy set for output variable.

6) Apply the appropriate defuzzification method to determine the value for output variable.

7) The output variable so obtained controls the system behaviour.

A system described by a numerical data which is highly non-linear, its equivalent fuzzy control system is based on its rule base. When a specific input is applied to the system it is first converted to fuzzy form. The input data is fuzzified i.e. Fuzzy membership functions are calculated corresponding to the input. The input may fall in various fuzzy sets of the input variable. Then its membership values for each fuzzy set are calculated. Similarly, the membership values are calculated for other input variables also. Initially, the rules with antecedents are established. Every rule established is labelled by its firing strength. Firing strength is calculated from the multiplication (OR operator) of the membership functions associated with fuzzy sets of the variables. In this way there will be many rules with different firing strengths are established for a specific input. The rule with maximum firing strength among the several rules is selected only. Similarly, for output data, when fuzzified, also falls in different fuzzy sets and will have different membership values in the respective fuzzy set. These membership values are combined by considering the maximum value (OR operator) of the membership in a fuzzy set. Next, weighted average defuzzification method is adopted to produce a single scalar value for each output variable. This approach combined with the genetic algorithm is followed for the air conditioning system example described in section $\mathrm{V}$.

\section{IV.GA-BASED OPTIMAL FUZZY LOGIC CONTROL:}

The Mamdani-type fuzzy rule system of eq. (1) with single output variable, in general, can be represented as;

$$
\begin{aligned}
& \text { If } x_{1} \text { is } A_{1} \text { and } x_{2} \text { is } A_{2} \text { and } \ldots \ldots x_{n} \text { is } A_{k} \\
& \text { then } y \text { is } B(\phi)
\end{aligned}
$$

where $\mathrm{y}$ is the output linguistic variables in the fuzzy set $B(\phi)$ and $\phi$ are supports of $B$ on which membership grade

$$
\mu_{B}(\phi)=1
$$

The weighted average defuzzification method is adopted, and then the control output of eq. (2) is given by

$$
y=\frac{\sum_{j=1}^{N} \phi^{j} \alpha_{j}}{\sum_{j=1}^{N} \alpha_{j}}
$$

.where $\alpha=\wedge \wedge_{i=1}^{n}\left(\mu_{A_{i}}\left(x_{i}\right)\right)$; the and operator $\wedge$ can

\begin{tabular}{|c|c|c|c|c|c|}
\hline S.No. & $\begin{array}{l}\text { Existing room } \\
\text { temp. }\left({ }^{\circ} \mathrm{C}\right)\end{array}$ & $\begin{array}{l}\text { Desired room } \\
\text { temp. }\left({ }^{\circ} \mathrm{C}\right)\end{array}$ & $\begin{array}{c}\text { Temp. Difference } \\
\left({ }^{\circ} \mathrm{C}\right)\end{array}$ & $\begin{array}{l}\text { Air flow rate } \\
\left(\mathrm{m}^{3} / \mathrm{sec}\right) \times 10^{-3}\end{array}$ & $\begin{array}{l}\text { Power consumed } \\
\text { (watts) }\end{array}$ \\
\hline 1 & 23.9 & 17.2 & 6.7 & 245 & 1660 \\
\hline 2 & 23.9 & 19.4 & 4.5 & 245 & 1670 \\
\hline 3 & 23.9 & 21.7 & 2.2 & 245 & 1690 \\
\hline 4 & 29.4 & 17.2 & 12.2 & 245 & 1820 \\
\hline 5 & 35 & 15 & 20 & 245 & 1950 \\
\hline 6 & 40.6 & 21.7 & 18.9 & 245 & 2320 \\
\hline 7 & 40.6 & 23.9 & 16.7 & 245 & 2360 \\
\hline 8 & 46.1 & 17.2 & 28.9 & 245 & 2350 \\
\hline 9 & 46.1 & 19.4 & 26.7 & 245 & 2580 \\
\hline 10 & 23.9 & 17.2 & 6.7 & 264 & 1670 \\
\hline 11 & 23.9 & 19.4 & 4.5 & 264 & 1690 \\
\hline 12 & 29.4 & 21.7 & 7.7 & 264 & 1870 \\
\hline 13 & 35 & 15 & 20 & 264 & 1970 \\
\hline 14 & 35 & 19.4 & 15.6 & 264 & 2020 \\
\hline 15 & 35 & 21.7 & 13.3 & 264 & 2050 \\
\hline 16 & 35 & 23.9 & 11.1 & 264 & 2080 \\
\hline 17 & 40.6 & 17.2 & 23.4 & 264 & 2260 \\
\hline 18 & 46.1 & 17.2 & 28.9 & 264 & 2520 \\
\hline 19 & 46.1 & 19.4 & 26.7 & 264 & 2550 \\
\hline 20 & 46.1 & 21.7 & 24.4 & 264 & 2590 \\
\hline
\end{tabular}
be 'min' or 'product'. $\mathrm{N}$ is the no. of rules and $\mathrm{n}$ is the number of input variables.

Table-2 Observed data of an air-conditioning system

Table-3 


\begin{tabular}{|c|c|c|c|c|}
\hline & S.No. & $\begin{array}{c}\text { Temp. Difference } \\
\left({ }^{\circ} \mathrm{C}\right)\end{array}$ & $\begin{array}{c}\text { Air flow } \\
\text { rate }\left(\mathrm{m}^{3} / \mathrm{sec}\right) \times 10^{-3}\end{array}$ & Power consumed (watts) \\
\hline & 1 & 2.2 & 245 & 1690 \\
\hline & 2 & 4.5 & 245 & 1670 \\
\hline & 3 & 6.7 & 245 & 1660 \\
\hline & 4 & 12.2 & 245 & 1820 \\
\hline & 5 & 16.7 & 245 & 2360 \\
\hline & 6 & 18.9 & 245 & 2320 \\
\hline & 7 & 20 & 245 & 1950 \\
\hline & 8 & 26.7 & 245 & 2580 \\
\hline & 9 & 28.9 & 245 & 2550 \\
\hline & 10 & 4.5 & 264 & 1690 \\
\hline & 11 & 6.7 & 264 & 1670 \\
\hline & 12 & 7.7 & 264 & 1870 \\
\hline & 13 & 11.1 & 264 & 2080 \\
\hline $\mathrm{O}$ & 14 & 13.3 & 264 & 2050 \\
\hline bvi & 15 & 15.6 & 264 & 2020 \\
\hline ousl & 16 & 20 & 264 & 1970 \\
\hline $\mathrm{y}$ & 17 & 23.4 & 264 & 2260 \\
\hline the & 18 & 24.4 & 264 & 2590 \\
\hline con & 19 & 26.7 & 264 & 2550 \\
\hline trol & 20 & 28.9 & 264 & 2520 \\
\hline
\end{tabular}

put of fuzzy rule based system depends on the parameters $\alpha_{j}$ and $\phi^{j}$. Hence, the genetic algorithms are applied towards adjusting the parameter

$\left\{\phi^{j}, \alpha_{j}\right\}, i=1,2, \ldots, n, j=1,2, \ldots, N \quad$ of $\quad$ eq. $\quad$ (3) so as to minimize the error function. The error function is dependent only on the difference between the obtained output and the target output

\section{AIR CONDITIONING SYSTEM MODEL DESIGN:}

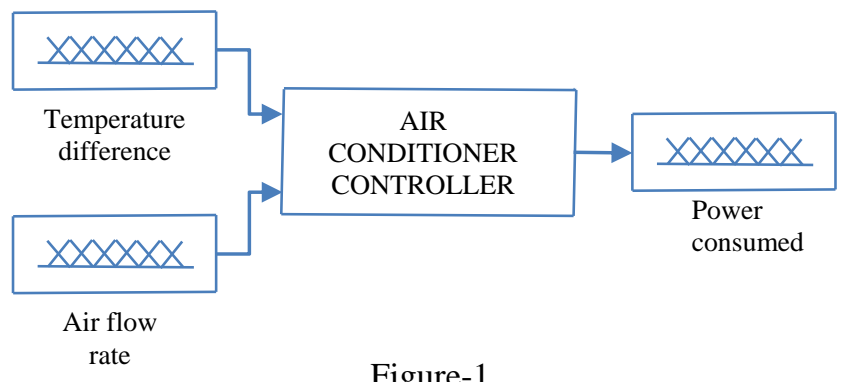

Figure-1

In this section we apply the GA-based optimal fuzzy logic control method, explained in section IV, to an air conditioning system model of figure-1. Table-2 shows the real time input-output observed data of the system. Following are the specifications of the system: Power source: $230 \mathrm{~V}, 50 \mathrm{~Hz}, 1-$ phase, Voltage Rating: $230 \mathrm{~V}(\mathrm{ac})$, Current rating: 10.2 A, Power input: $2240 \mathrm{~W}$, cooling tones: 2. Our aim is to obtain a compact and optimal fuzzy rulebased model. (a)

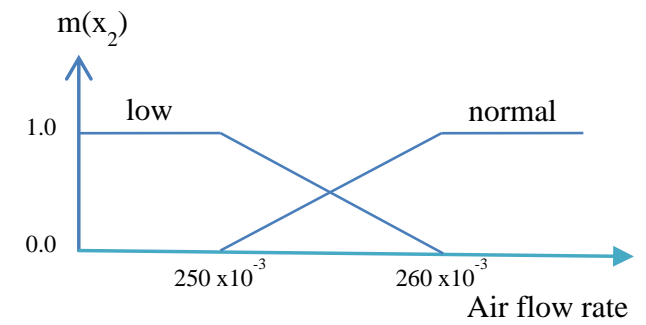

(b)

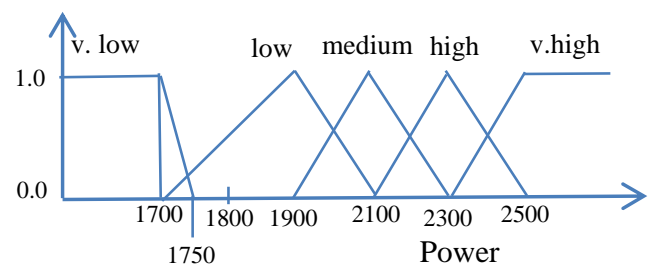

(c)

Figure-2 Division of the input and output spaces into fuzzy regions and the corresponding membership functions. (a) $\mathrm{m}\left(\mathrm{x}_{1}\right)$ (b) $\mathrm{m}\left(\mathrm{x}_{2}\right)$ (c) $\mathrm{m}(\mathrm{y})$.

We first identify the input and output variables. We chose temperature difference $(\Delta T)$ and airflow rate as the system input and power consumed as output variables. The variable temperature difference $(\Delta T)$ is calculated as the difference between the existing room temperature and the desired room temperature of table- 2 . Thus table- 2 is redrawn with above change as table-3. Thus, the system has two inputs and a single output as shown in figure- 1 .

\subsection{Fuzzy sets:}


It is observed from table-3 that input variables temperature difference varies from $2^{0} \mathrm{C}$ to $29^{\circ} \mathrm{C}$, airflow rate is either $264 \times 10^{-3} \mathrm{~m}^{3} / \mathrm{sec}$. or $245 \times 10^{-}$ ${ }^{3} \mathrm{~m}^{3} / \mathrm{sec}$ and output variable power consumed ranges from 1660 watts to 2590 watts. Depending upon the variations in the value of each variable and experience the number of fuzzy sets and shapes of the membership functions associated with each fuzzy set are chosen. Triangular shape of membership function for both input and output variables of the fuzzy system is adopted. Whereas, the no. of fuzzy sets is concerned, theoretically, each fuzzy variable can have many fuzzy sets with each having its own membership function, but commonly used are three, five, seven or nine fuzzy sets for each fuzzy variable. In our case, the input variable temperature difference has three fuzzy sets representing the linguistic descriptions: low, medium and high. The second input variable i.e. airflow rate has two fuzzy sets with linguistic descriptions: low and normal. The output variable i.e. power consumed by the air-conditioner controller has five fuzzy sets labelled as: very low, low, medium, high and very high. For simplicity for the output we assume only four fuzzy sets labelled by; very low, medium, high and very high. Therefore, two bits are sufficient to represent the four states of consequent part. Table- 5 shows the binary codes and its equivalent real values for each output fuzzy set.

The fuzzy sets which characterize the inputs and output variables are given in fig. 2.

\subsection{Fuzzy Rule base:}

For the said fuzzy system, the rule base is as shown in table-4.

Table-4

Rule 1: If (temperature difference $(\Delta \mathrm{T})$ is 'low') and (airflow rate is 'low') then (power consumed is ......................)

Rule 2: If (temperature difference $(\Delta \mathrm{T})$ is 'low') and (airflow rate is 'normal') then (power consumed is ......................)

Rule 3: If (temperature difference $(\Delta \mathrm{T})$ is 'medium') and (airflow rate is 'low') then (Power consumed is

Rule 4: If (temperature difference $(\Delta \mathrm{T})$ is 'medium') and (airflow rate is 'normal') then (power consumed is ...)

Rule 5: If (temperature difference $(\Delta \mathrm{T})$ is 'high') and (airflow rate is 'low') then (power consumed is

Rule 6: IF (temperature difference $(\Delta \mathrm{T})$ is 'high') and (airflow rate is 'normal') then (power consumed is ......................)

\subsection{Fuzzification of Inputs:}

For fuzzification of inputs, that is to compute the fuzzy membership values for the antecedents, we consider any one set of measured value of input from observed data of table-3 at a time. For example, let the measured temperature difference be $16^{\circ} \mathrm{C}$ and the air flow rate be $245 \times 10^{-3} \mathrm{~m}^{3} / \mathrm{sec}$ taken from the said table.

The input temperature difference deals with 3 fuzzy sets namely low, medium and high. For temperature difference of $16^{\circ} \mathrm{C}$, the fuzzy membership values are computed graphically in figure- 3 .

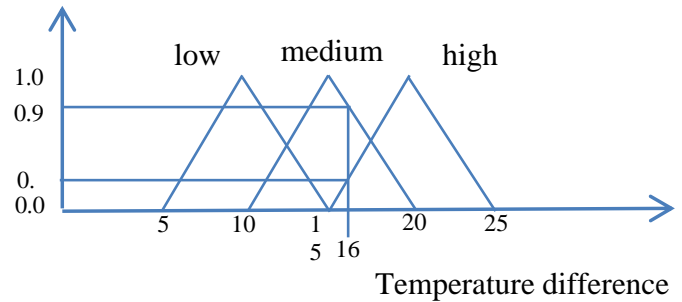

Figure-3

The fuzzy membership value in fuzzy sets medium and high comes out to 0.1 and 0.9 respectively. Whereas the value $16^{\circ} \mathrm{C}$ does not fall in the range of fuzzy set low, so its fuzzy membership value in this set is taken as zero.

Similarly, for air flow rate $\left(=245 \times 10^{-3} \mathrm{~m}^{3} / \mathrm{sec}\right)$ the qualifying fuzzy sets are shown in figure- 4 .

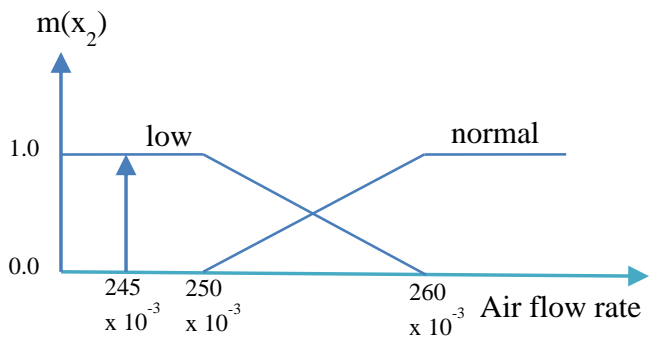

Figure-4

The fuzzy membership function of air flow rate $=245 \times 10^{-3} \mathrm{~m}^{3} / \mathrm{sec}$ for set low is $\mu_{\text {low }}(x)=1$ and for fuzzy set high is $\mu_{h i g h}(x)=0$.

\subsection{Computation of Rule Strength:}

The rule strength $\left(\alpha_{i}\right)$ associated with $i^{\text {th }}$ rule is obtained by computing the minimum of the membership functions of the antecedents, given by;

$\alpha_{i}=\min .[\mu($ temp. difference $), \mu($ air flow rate $)]$ 
For the rule base of table- 4 the rule strengths for each rule are calculated as;

Rule1: $\alpha_{1}=$

$\min .\left(\mu_{\text {low }}\right.$ (temp. difference $), \mu_{\text {low }}($ air flow rate $\left.)\right)$

$=\min \cdot(0,1)=0$

Rule 2: $\alpha_{2}=$

$\min .\left(\mu_{\text {low }}(\right.$ temp. difference $), \mu_{\text {normal }}($ air flow rate $\left.)\right)$

$=\min .(0,0)=0$

Rule 3: $\alpha_{3}=$

$\min .\left(\mu_{\text {medium }}(\right.$ temp. difference $), \mu_{\text {low }}($ air flow rate $\left.)\right)$

$=\min \cdot(0.9,1)=0.9$

Rule 4: $\alpha_{4}=$

$\min .\left(\mu_{\text {medium }}(\right.$ temp. difference $), \mu_{\text {normal }}($ air flow rate $\left.)\right)$

$=\min \cdot(0.9,0)=0$

Rule 5: $\alpha_{5}=$

$\min .\left(\mu_{\text {high }}(\right.$ temp. difference $), \mu_{\text {low }}($ air flow rate $\left.)\right)$

$=\min \cdot(0.1,1)=0.1$

Rule6: $\alpha_{6}=$

$\min .\left(\mu_{\text {high }}(\right.$ temp. difference $), \mu_{\text {normal }}($ air flow rate $\left.)\right)$

$=\min \cdot(0.1,0)=0$

The values of $\alpha_{1}$ to $\alpha_{6}$ are found to;

$$
\begin{aligned}
& {\left[\begin{array}{llllll}
\alpha_{1} & \alpha_{2} & \alpha_{3} & \alpha_{4} & \alpha_{5} & \alpha_{6}
\end{array}\right]=} \\
& {\left[\begin{array}{llllll}
0 & 0 & 0.9 & 0 & 0.1 & 0
\end{array}\right]}
\end{aligned}
$$

\subsection{Implementing GA:}

Each rule in the rule base of table- 4 has only the information about the antecedent part, whereas the consequent part is missing. The GA is applied in the present work to find the consequent part for each of the rule to complete the rule base and formation of an optimal rule base. Each data set produces a rule automatically under this approach. The GA is applied in the following steps;

(a) Representation/Encoding:

Consider a chromosome given as;

$$
\begin{array}{llllllllllll}
0 & 0 & 0 & 0 & 0 & 1 & 0 & 0 & 1 & 0 & 0 & 1
\end{array}
$$

Starting from the left hand side, bit number 1 and 2 shows 00 binary code and represent the fuzzy set ' $\mathrm{v}$. low' for consequent of the rule in the rule base. Similarly, each bit pair represents one fuzzy set of the output along with its equivalent real number value is shown by table- 5 .

Table-5

\begin{tabular}{|c|c|c|}
\hline $\begin{array}{c}\text { Fuzzy set } \\
\text { of output }\end{array}$ & Binary code & $\begin{array}{c}\text { Equivalent real } \\
\text { value }\end{array}$ \\
\hline v.low & 00 & 0 \\
\hline normal & 01 & 1 \\
\hline
\end{tabular}

\begin{tabular}{|c|c|c|}
\hline high & 10 & 2 \\
\hline v.high & 11 & 3 \\
\hline
\end{tabular}

(b) Generation of chromosomes:

Initially a population of four chromosomes are generated randomly. Suppose for gen $=0$ initial population of chromosomes are;

$\begin{array}{llllllllllll}0 & 0 & 0 & 0 & 0 & 1 & 0 & 0 & 1 & 0 & 0 & 1 \\ 1 & 1 & 0 & 0 & 1 & 0 & 0 & 0 & 0 & 0 & 1 & 0 \\ 1 & 0 & 0 & 0 & 1 & 1 & 1 & 0 & 0 & 0 & 1 & 1 \\ 0 & 0 & 1 & 0 & 1 & 0 & 1 & 1 & 0 & 0 & 0 & 1\end{array}$

For example, considering the first chromosome only from the initial population;

$\begin{array}{llllllllllll}0 & 0 & 0 & 0 & 0 & 1 & 0 & 0 & 1 & 0 & 0 & 1\end{array}$

Table- 6 describes each bit pair of the above chromosome starting from left to right.

Table-6

\begin{tabular}{|c|c|c|c|}
\hline $\begin{array}{c}\text { Bits } \\
\text { position }\end{array}$ & $\begin{array}{c}\text { Binary } \\
\text { code }\end{array}$ & $\begin{array}{c}\text { Equivalent } \\
\text { real no. }\end{array}$ & $\begin{array}{c}\text { Denoted } \\
\text { by }\end{array}$ \\
\hline $1 \& 2$ & 00 & 0 & $\phi^{1}$ \\
\hline $3 \& 4$ & 00 & 0 & $\phi^{2}$ \\
\hline $5 \& 6$ & 01 & 1 & $\phi^{3}$ \\
\hline $7 \& 8$ & 00 & 0 & $\phi^{4}$ \\
\hline $9 \& 10$ & 10 & 2 & $\phi^{5}$ \\
\hline $11 \& 12$ & 01 & 1 & $\phi^{6}$ \\
\hline
\end{tabular}

(c) Fitness function:

The next important consideration following the representation is the choice of the fitness function. The fitness function measures the performance of the system. We used absolute difference error function as fitness function for the air conditioning controller system and is defined by;

Error $=\mathrm{E}=($ obtained output - target output $)$

The output is calculated for each chromosome as the weighted average of the rule strengths for each rule and is given

$y=\frac{\alpha_{1} \phi^{1}+\alpha_{2} \phi^{2}+\alpha_{3} \phi^{3}+\alpha_{4} \phi^{4}+\alpha_{5} \phi^{5}+\alpha_{6} \phi^{6}}{\alpha_{1}+\alpha_{2}+\alpha_{3}+\alpha_{4}+\alpha_{5}+\alpha_{6}}$ by,

Substituting the values of rule strengths $\alpha_{i}$ as computed earlier and values of $\phi^{i}$ ( considering only first chromosome from the initially generated population) from table-6, output is found to be, $y=1.1$. Then the fitness function denoted as error from equation (4) is found to be; Error $=0.9$. Similarly, the fitness values are calculated for other chromosomes of the population.

New populations of chromosomes are generated using reproduction, crossover and mutation operators as explained earlier and their fitness is evaluated. Fittest individuals are retained and rest are discarded. 
(d) Termination of GA:

The GA is terminated when the chromosomes with fitness function value satisfies the following condition;

$$
\text { | error } \mid \leq 0.1
$$

The whole problem can also be stated, alternatively, as; Determine the values of $\phi^{1}, \phi^{2}, \ldots \ldots, \phi^{6}$ from equation (5) such that error condition of (6) is satisfied. where $\phi^{j} \in\{v$. low, normal, high, v. high $\}$.

These four states are represented by the binary codes and the equivalent real values shown in table5 .

\section{SIMULATION AND DISCUSSION:}

Table -7 Genetic Algorithm parameters used

\begin{tabular}{|c|c|c|}
\hline Sr. No. & Parameter & Value \\
\hline 1 & Gene size & 2 \\
\hline 2 & Number of rules & 6 \\
\hline 3 & Chromosome size & 12 \\
\hline 4 & $\begin{array}{c}\text { Number of } \\
\text { chromosomes in a } \\
\text { population }\end{array}$ & 4 \\
\hline 5 & Mutation rate & 0.0375 \\
\hline 6 & Crossover rate & 0.7 \\
\hline 7 & Stopping Criterian & error $\mid \leq 0.1$ \\
\hline 8 & Type of reproduction & $\begin{array}{c}\text { Stochastic } \\
\text { universal } \\
\text { sampling }\end{array}$ \\
\hline 9 & Type of mutation & Discrete mutation \\
\hline 10 & Type of crossover & $\begin{array}{c}\text { Single point } \\
\text { crossover }\end{array}$ \\
\hline
\end{tabular}

The implementation of the genetic algorithm is written in Matlab. The optimal rule base has been evolved for the air conditioning system example, which consists of real time measurements of the system. It consists of 20 instances with two inputs and a single output attributes i.e. temperature difference (input 1), air flow rate (input 2) and power consumed (output) as recorded in table-2 and table-3. We considered three fuzzy sets for the first input variable, two fuzzy sets for $2^{\text {nd }}$ input variable and four fuzzy sets for the output variable with linear triangular membership functions. The fuzzy system was evolved with the membership functions are fixed as per figure- 2 and the crossover rate and mutation rates were held constant during the run as per table-7 which also shows the other GA parameters used during run for the fuzzy model evaluation.

In all experiments, the number of iterations needed to reach the specified limit of error $(\leq 0.1)$ was determined for each run is shown by the bar graphs of figure 5 and figure 6 . The performance of the GA at each iteration is shown below;

Performance of GA

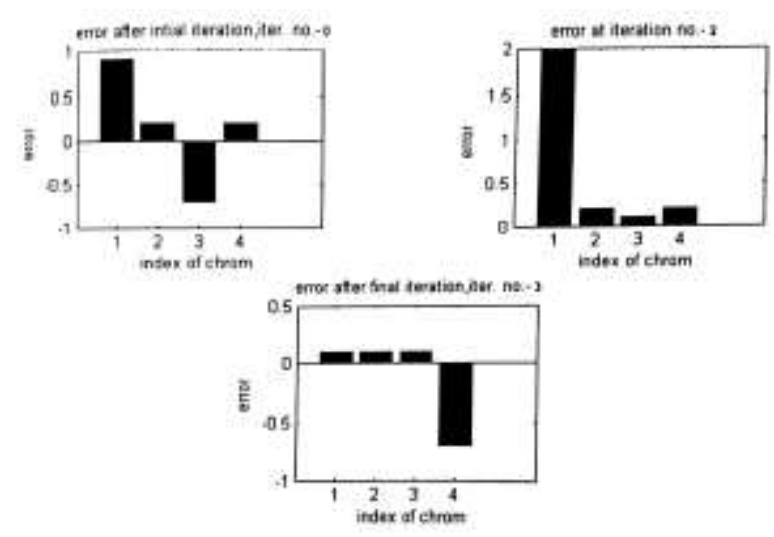

Figure-5
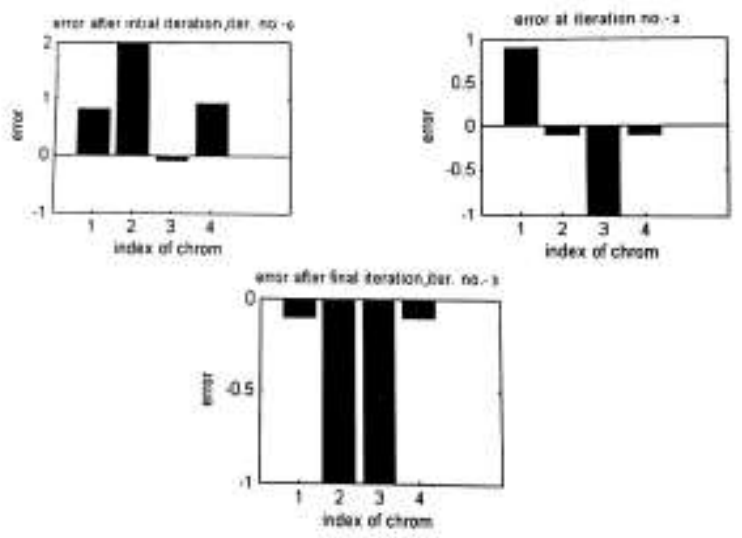

Figure-6

The optimal fuzzy rules so generated are listed below;

- If (temperature difference $(\Delta \mathrm{T})$ is 'low') and (airflow rate is 'low') then (power consumed is 'v. low')

- If (temperature difference $(\Delta \mathrm{T})$ is 'low') and (airflow rate is 'normal') then (power consumed is 'v. low')

- If (temperature difference $(\Delta \mathrm{T})$ is 'medium') and (airflow rate is 'low') then (Power consumed is 'high'.)

- If (temperature difference $(\Delta \mathrm{T})$ is 'medium') and (airflow rate is 'normal') then (power consumed is 'medium')

- If (temperature difference $(\Delta \mathrm{T})$ is 'high') and (airflow rate is 'low') then (power consumed is 'v. high')

- If (temperature difference $(\Delta \mathrm{T})$ is 'high') and (airflow rate is 'normal') then (power consumed is ' $\mathrm{v}$. high')

\section{CONCLUDING REMARKS:}

In earlier work [16] the fuzzy rule base from the numerical data pairs is evolved with the example of an air conditioning system by utilizing the Wang and Mendel [3] technique. The proposed GA methodology has following advantages over the conventional methods; 
The proposed technique is a general procedure for generation of fuzzy rules from the numerical data. Time consumed is very low as compared to the conventional methods. There is lot of freedom in choosing the membership functions, this provides us with lot of flexibilities of designing a system according to different requirements.

The suggested work can be extended, by adding more parameters, to find; Number of membership functions for each variable, type of membership function, number of rules. Other evolutionary algorithms such as particle swarm optimizers can be used instead of a GA.

Future work could be to investigate the influence of increased size of chromosomes, changing various parameters of the algorithm like mutation rate, crossover rate with an objective to improve the performance further. The approach can be used for finding the best parameters of GA for any system under consideration with less number of iterations and hence little computational time and improved performance.

\section{REFERENCES:}

[1] L. A. Zadeh, 'Fuzzy sets:' Information and Control, vol. 8, no. 3, pp. 338-353, 1965.

[2] L. A. Zadeh, "Fuzzy algorithms," Information and Control, vol. 12, no. 2, pp. 94-102, 1968.

[3] Li-Xin Wang and Jerry M. Mendel, "Generating fuzzy rules by learning from examples" IEEE transactions on Systems, Man and Cybernatics, Vol.-22, no.-6, pp. 1414-1427, Nov./Dec- 1992.

[4] Magne Setnes, "Supervised Fuzzy Clustering for Rule Extraction", IEEE Transactions on Fuzzy systems, Vol.-8, No.-4, pp. 416-424, August 2000.

[5] Shyi-Ming Chen and Fu-Ming Tsai, "A New Method to Construct Membership Functions and Generate Fuzzy Rules from Training Instances", Information and Management Sciences, Vol-16, no.-2, pp. 47-72, June, 2005.

[6] J.L. Castro, J.M. Zurita, "An inductive learning algorithm in fuzzy systems", Fuzzy Sets and Systems, 89 (1997), 193-203.

[7] J.L. Castro, J.J. Castro-Schez and J.M. Zurita, "Learning maximal structure rules in fuzzy logic for knowledge acquisition in expert systems" , Fuzzy Sets and Systems, 101 (1999), 331-342.
[8] Tzung-Pei Hong and Chai-Ying Lee, "Induction of fuzzy rules and membership functions from training examples", Fuzzy Sets and Systems, 84 (1996), 33-47.

[9] Tzung-Pei Hong and Jyh-Bin Chen, "Finding relevant attributes and membership functions", Fuzzy Sets and Systems, 103 (1999), 389-404.

[10] K. De Jong, “Adaptive system design: a genetic approach", IEEE Trans. System, Man, Cybern., vol. 10, no. 9, pp. 566-574, Sep. 1980.

[11] D.E. Goldberg, "Genetic Algorithms in Search, Optimization and Machine Learning”, Reading, MA: Addision-Wesley, 1989.

[12] C.L. Karr and E.J. Gentry, "Fuzzy control of $\mathrm{pH}$ using genetic algorithms," IEEE Transactions on Fuzzy Systems, Vol. 1, no. 1, pp. 46-53, Feb. 1993.

[13] C.L. Karr, "Design of an adaptive fuzzy logic controller using a genetic algorithm." In Proc. The Fourth Int. Conf. Genetic Algorithms.1991, pp. 450-57.

[14] Abdollah Homaifar and Ed McCormick, "Simultaneous Design of Membership Functions and Rule Sets for Fuzzy Controllers Using Genetic Algorithms," IEEE Transactions on Fuzzy systems, Vol.-3, No.-2, pp. 129-138, May 1995.

[15] Yuhui Shi, Russel Eberhart and Yaobin Chen, "Implementation of Evolutionary Fuzzy Systems," IEEE Transactions on Fuzzy systems, Vol.-7, No.2, pp. 109-119, April 1999.

[16] Sanjay Charaya, "Generating fuzzy rules from numerical data", International journal of latest trends in Engineering \& Technology. Vol. 18, issue 2, pp. 013-019.

\section{AUTHORS PROFILE}




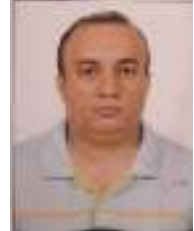

Sanjay Charaya is working as an Assistant Professor in Electrical engineering under the Department of Electrical Engineering, CDL State Institute of Engineering \& Technology, PanniwalaMota, Sirsa, India. His research interests are in the field of non-linear control, fuzzy systems, genetic algorithm, intelligent control \& systems and optimal control.
Kapil Mehta is working as an Assistant Professor in the Department of Computer Science and Engineering, Landran, Mohali, Punjab, India. His research interests are Wireless Sensor Networks, intelligent systems, smart homes, activity recognition, and healthcare systems. $\mathrm{He}$ has facilitated innumerable executive team planning sessions. $\mathrm{He}$ has $30+$ publications in reputed International Journals and filed 20 Patents in India till date. He has spoken to various enterprise managers and information professionals for conducting various events in Club Chapters like IEEE, IETE and IEI etc.

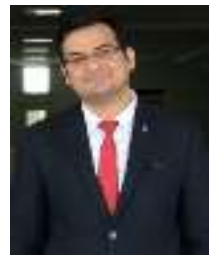

